\title{
Douleur thoracique atypique révélatrice d'un kyste pleuropéricardique
}

\section{Atypical Thoracic Pain Revealing a Pleuropericardial Cyst}

\author{
P.-N. Claeys · V. Mazy \\ Reçu le 27 mars 2019; accepté le 27 juin 2019 \\ (C) SFMU et Lavoisier SAS 2019
}

\section{Introduction}

Le kyste pleuropéricardique est une anomalie embryonnaire rare dont la prévalence est estimée à 1/100 000 [1] et est une cause rare de douleur thoracique atypique. Ces kystes sont le plus souvent asymptomatiques mais peuvent entraîner : de la dyspnée, une insuffisance cardiaque droite secondaire à la compression, une pneumopathie [1], une tamponnade cardiaque, un choc cardiogénique, un prolapsus de la valve mitrale, une fibrillation auriculaire, une obstruction, une hémorragie interne spontanée, une sténose pulmonaire et une mort subite [2]. Face à ces symptômes aspécifiques pouvant évoquer de nombreuses pathologies, il est intéressant de ne pas oublier l'évocation de cette pathologie au niveau du diagnostic différentiel. Nous rapportons ici le cas d'une patiente de 35 ans consultant aux urgences pour une douleur thoracique atypique de survenue spontanée dont le diagnostic final est celui d'un kyste pleuropéricardique.

\section{Observation}

Il s'agit d'une patiente de 35 ans se présentant aux urgences pour une douleur rétrosternale respirodépendante avec dyspnée positionnelle, survenue spontanément vers $4 \mathrm{~h}$ du matin. Ses antécédents comportaient : un by-pass gastrique et un reflux gastro-œsophagien (traité par du pantoprazole $20 \mathrm{mg}$ ), de l'endométriose et un goitre. Lors de son admission aux urgences, la patiente est hypertendue $(170 / 72 \mathrm{mmHg})$, afébrile. Sa saturation en oxygène est de $98 \%$. À l'anamnèse la patiente décrit une douleur non irradiante, ressentie comme un coup de poignard et non soulagée par la prise d'ibuprofène $400 \mathrm{mg}$. La douleur est majorée

P.-N. Claeys $(\bowtie) \cdot$ V. Mazy

Service des urgences, CHR Val-de-Sambre, rue Chère-Voie 75, B-5060 Auvelais, Belgique

e-mail : pniels.claeys@chrsm.be lorsqu'elle se trouve en décubitus dorsal latéral droit, et une amélioration des douleurs est observée lorsqu'elle se trouve en position assise. À l'examen clinique, l'auscultation cardiopulmonaire ne révèle pas d'anomalie, la palpation du thorax met en évidence une sensibilité au niveau des articulations sternocostales. L'abdomen est sans particularité, hormis une légère sensibilité à la palpation épigastrique. La prise de sang effectuée ne révèle pas d'anomalie (troponine $\mathrm{T} \mathrm{HS}<5 \mathrm{ng} / \mathrm{l})$. L'électrocardiogramme montre un rythme sinusal régulier à $80 / \mathrm{min}$ sans autre anomalie. $\mathrm{La}$ radiographie du thorax met en évidence une opacité floue au niveau basal droit (Fig. 1A). Devant ce tableau de douleur thoracique atypique avec troponine négative, la réalisation d'une échographie cardiaque transthoracique et celle d'un scanner thoracique (non injecté) ont été effectuées. L'échographie cardiaque transthoracique met en évidence une image de matériel hétérogène en regard de l'oreillette droite dont l'aspect semble liquidien. Le scanner thoracique non injecté révèle un kyste pleuropéricardique mesurant entre 7 et $9 \mathrm{~cm}$ de grand axe au niveau du sinus cardiophrénique droit (Fig. 1B) ainsi qu'un petit foyer de comblement alvéolaire au niveau du lobe moyen, conséquence de phénomènes compressifs dus à ce kyste pleuropéricardique volumineux. Devant ce tableau de kyste pleuropéricardique symptomatique associant douleur thoracique, dyspnée positionnelle et pneumopathie par compression du lobe moyen, une résection a été pratiquée par thoracoscopie (Fig. 1C). En peropératoire, après marsupialisation du kyste, un pertuis entre le péricarde et la plèvre pariétale médiastinale a été découvert confirmant le diagnostic de kyste pleuropéricardique. Les suites opératoires ont été simples avec un drain thoracique retiré trois jours après l'opération et une disparition des douleurs ainsi que de la dyspnée. La patiente est sortie de l'hôpital cinq jours après l'opération.

\section{Discussion}

Le kyste pleuropéricardique est une tumeur bénigne du médiastin secondaire à une anomalie de cloisonnement des 

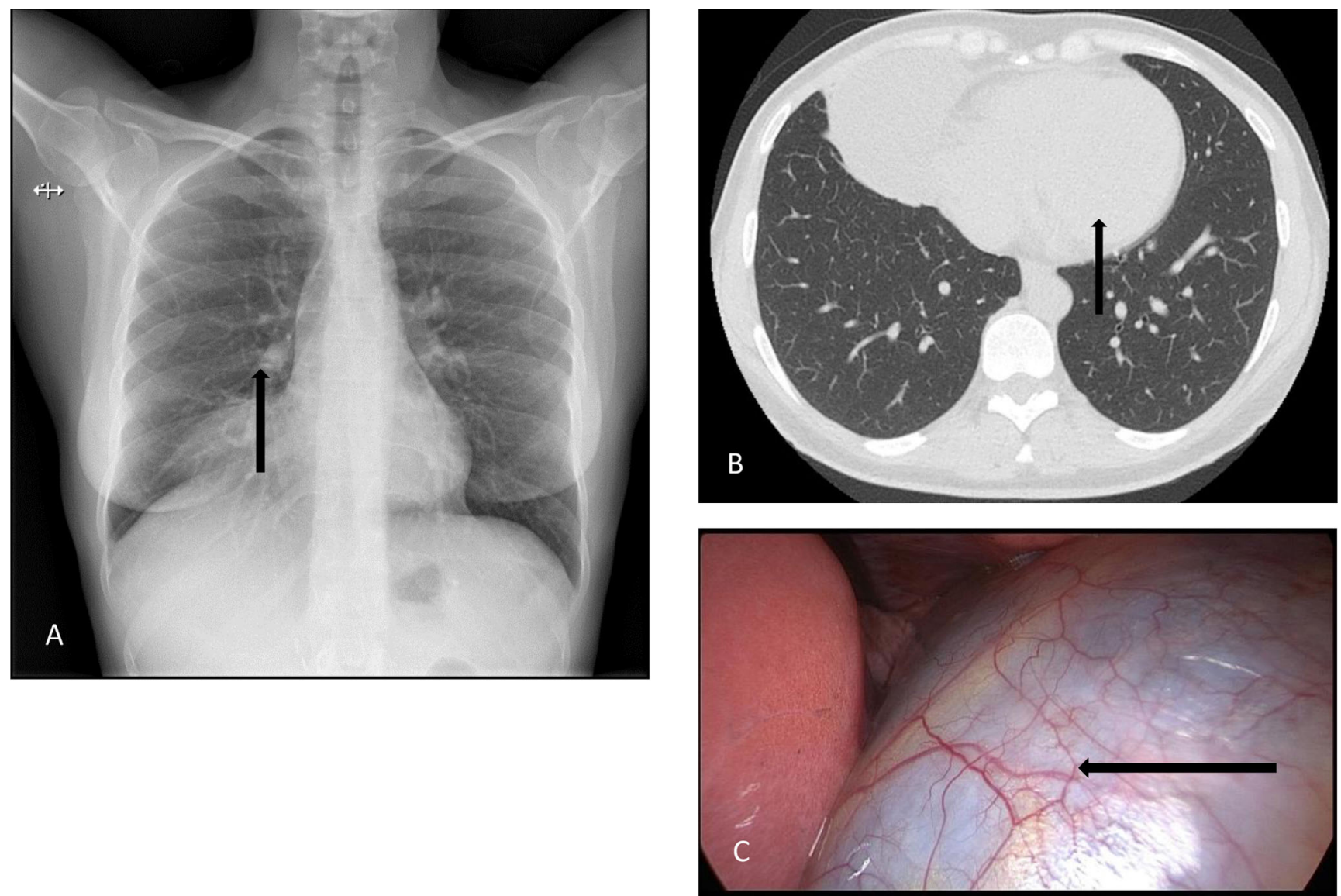

Fig. 1 A. Radiographie thoracique montrant une opacité floue au niveau de la base droite (flèche). B. Scanner thoracique non injecté montrant le kyste pleuropéricardique (flèche). C. Photographie peropératoire du kyste pleuropéricardique (flèche)

cavités cœlomiques [3]. Les kystes pleuropéricardiques représentent $7 \%$ de toutes les tumeurs du médiastin et $11 \%$ des kystes médiastinaux dont $70 \%$ se situent au niveau de l'angle cardiophrénique droit [4]. L'échographie cardiaque transthoracique n'est pas indispensable au diagnostic. Cependant, si celle-ci est réalisée par l'urgentiste, elle oriente plus rapidement le diagnostic, ce qui permet un gain de temps conséquent et l'exclusion d'autres étiologies telles qu'une péricardite, un choc cardiogénique ou un infarctus. Le diagnostic est suggéré par le scanner thoracique qui met en évidence une masse liquidienne en contact du péricarde, mais ne devient définitif qu'en peropératoire ou après analyse anatomopathologique [5].

Les situations rares devant faire penser à ce diagnostic sont à évoquer lorsqu'il persiste une douleur non soulagée par des antalgiques, des symptômes évoquant une compression intrinsèque, ainsi que nous l'évoquions dans l'introduction, et ce, malgré un bilan biologique et électrocardiographique initial négatif. Ces manifestations cliniques peuvent être : une dyspnée, une pneumopathie par compression, une insuffisance cardiaque droite secondaire à la compression, une tamponnade cardiaque, un choc cardiogénique, un prolapsus de la valve mitrale, une fibrillation auriculaire, une obstruction, une hémorragie interne spontanée, une sténose pulmonaire et une mort subite.

Le traitement des formes asymptomatiques consiste en une surveillance simple du kyste qui ne doit être opéré qu'en cas de complications [2]. L'exérèse n'est indiquée que pour les kystes volumineux symptomatiques [6] et doit être complète afin d'éviter les récidives. En cas d'opération, la voie à privilégier est la vidéothoracoscopie, car elle diminue les séquelles postopératoires [7].

En conclusion, l'existence d'une douleur thoracique position-dépendante associée à des symptômes pouvant être dus à une compression intrinsèque doit faire évoquer le diagnostic de kyste pleuropéricardique. En raison des complications que cette pathologie peut occasionner, il est important de ne pas méconnaître ce diagnostic rare.

Liens d'intérêts : les auteurs déclarent ne pas avoir de liens d'intérêts. 


\section{Références}

1. Berry MF (2018) Approach to the adult with a mediastinal mass, uptodate. https://www.uptodate.com/contents/approach-to-the-adultpatient-with-a-mediastinal-mass?search=Approach $\% 20$ to $\% 20$ the $\% 20$ adult $\% 20$ patient $\% 20$ with $\% 20 \mathrm{a} \% 20$ mediastinal $\% 20$ mass\&source $=$ search result\&selectedTitle $=1 \sim 150 \&$ usage type $=$ default\&display rank $=1$ (Dernier accès le 24 janvier 2019)

2. Ondo N'dong F, Mwanyombet L, Bayonne L, et al (1995) Calcified pleuropericardic cysts -2 cases report. West Afr J Med 14:65-8

3. Orphanet, le portail des maladies rares et des médicaments orphelins, kystes pleuropéricardiques. https://www.orpha.net/consor/ cgi-bin/OC_Exp.php?lng=FR\&Expert=99131 (Dernier accès le 13 mars 2019)
4. Asriri A, Mahmal A, Tounsaoui B (2004) Le kyste pleuropéricardique, à propos d'un cas. Rev Mal Respir 21:1S58-104

5. Chataigner O, Gossot D, Validire P, et al (2001) Abord des kystes pleuropéricardiques par médiastinoscopie. Rev Mal Respir 18:66-7

6. Le Pimpec-Barthes F, Cazes A, Bagan A, et al (2010) Les kystes du médiastin : approche diagnostique et traitement. Rev Pneumol Clin 66:52-62

7. Demmy T, Dexter E (2017) Overview of minimally invasive thoracic surgery, uptodate. https://www.uptodate.com/contents/overviewof-minimally-invasive-thoracic-surgery? search $=$ overview $\% 20 \mathrm{of} \% 20$ minimally $\% 20$ invasive $\% 20$ thoracic $\% 20$ surgery\&source $=$ search result\&selectedTitle $=1 \sim 150 \&$ usage_type $=$ default\&display_rank $=1$ (Dernier accès le 27 mars 2019) 